\title{
COVID-19 infection during pregnancy - maternal and perinatal outcomes: a tertiary care centre study
}

\author{
Nazia Hassan', Mudasir Muzamil2*, Dawood Banday ${ }^{3}$
}

\begin{abstract}
${ }^{1}$ Department of Obstetrics and Gynecology, SKIMS, Medical College and Hospital, Srinagar, Jammu and Kashmir, India

${ }^{2}$ Department of Cardiology, SKIMS Soura Srinagar, Jammu and Kashmir, India

${ }^{3}$ Department of Medicine, SKIMS, Soura, Srinagar, Jammu and Kashmir, India
\end{abstract}

Received: 30 June 2020

Accepted: 10 August 2020

\section{*Correspondence:}

Dr. Mudasir Muzamil,

E-mail: drmmuzamil@gmail.com

Copyright: ( ) the author(s), publisher and licensee Medip Academy. This is an open-access article distributed under the terms of the Creative Commons Attribution Non-Commercial License, which permits unrestricted non-commercial use, distribution, and reproduction in any medium, provided the original work is properly cited.

\section{ABSTRACT}

Background: With the exponential increase in the severe acute respiratory syndrome coronavirus 2 (SARS-CoV2/COVID-19) worldwide, an increasing proportion of pregnant women are now infected during their pregnancy. The knowledge gained from previous human coronavirus outbreaks suggests that pregnant women and their fetuses are particularly susceptible to poor outcomes. The aim of this study was to summarize the maternal and perinatal outcomes of pregnant women infected with COVID-19 during their pregnancy.

Methods: Eligibility criteria included pregnant women positive for COVID-19 as detected by real-time polymerase chain reaction (PCR) or dual fluorescence PCR-confirmed SARS-CoV-2 infection.

Results: A total of 38 pregnant women positive for COVID-19 as confirmed by RT-PCR, were included in the study. $21 \%$ cases had preterm deliveries and LSCS was the preferred mode of delivery in 23 of 38 i.e., $60.5 \%$ cases. There was a tendency for low Apgar score at birth, higher rates of fetal distress, meconium, NICU admissions. There was one IUD and one neonatal death. The treatment patterns of COVID-19 infection among pregnant women during their pregnancy or following delivery was mostly supportive in the form of oxygen and antibiotic therapy.

Conclusions: Although the majority of mothers were discharged without any major complications, severe maternal morbidity as a result of COVID-19 and perinatal deaths were reported. Vertical transmission of the COVID-19 could not be ruled out. Pregnant infected women had different symptoms, and they were given mostly supportive treatments than the general infected population. Careful monitoring of pregnancies with COVID-19 and measures to prevent neonatal infection are warranted.

Keywords: Coronavirus-2019, COVID-19, Polymerase chain reaction, Pregnancy, Pregnant women

\section{INTRODUCTION}

In the beginning of December 2019, a cluster of persons with a pneumonia of unknown cause was identified in Wuhan, the capital of Hubei province and a large city of approximately 11 million persons located in the central region of the People's Republic of China. ${ }^{1,2}$ On $7^{\text {th }}$ January 2020 investigators in China identified the etiological agent of the epidemic as a previously unknown coronavirus, and it was given the designation 2019-nCoV (for 2019 novel Coronavirus). ${ }^{2}$ With over a million individuals infected, the global pandemic caused by the severe acute respiratory syndrome coronavirus 2 (SARS-CoV-2) has been growing at an accelerating rate. The increasing mortality rate warrants identification and protection of the vulnerable populations in society. The knowledge gained from previous human coronavirus outbreaks, namely, the severe acute respiratory syndrome 
coronavirus (SARS-CoV) and the middle East respiratory syndrome coronavirus (MERS-CoV), suggests that pregnant women and their fetuses are particularly susceptible to poor outcomes. Admission to intensive care is common and a case fatality rate of up to $35 \%$ has been documented. ${ }^{3,4}$

The physiological changes occurring during pregnancy make the mother more vulnerable to severe infections. Anatomical changes such as an increase in the transverse diameter of the thoracic cage and an elevated level of the diaphragm, decrease maternal tolerance to hypoxia. ${ }^{5}$ Lung volume changes and vasodilation can lead to mucosal edema and increased secretions in the upper respiratory tract. In addition, alterations in cell-mediated immunity contribute to the increased susceptibility of pregnant women to be infected by intracellular organisms such as viruses. With regard to the fetus and the newborn, the immaturity of the innate and adaptive immune systems makes them highly susceptible to infections. Dysregulation of factors such as cytokines and the complement cascade can have deleterious consequences for brain development and function. ${ }^{6}$ Pregnant women and their new-borns should be evaluated for being potential risk groups in the current COVID-19 pandemic.

On $5^{\text {th }}$ February 2020 it was reported by multiple media outlets that a new-born infant delivered during the epidemic in Wuhan had tested positive for 2019-nCoV at the Wuhan Children's Hospital in Hubei province 30 hours following its birth. Reports have stated that the infant had stable vital signs, no fever or cough, but had shortness of breath together with abnormal chest radiographs and abnormalities of liver function. ${ }^{7-9} \mathrm{Dr}$. Zeng Lingkong, chief physician at the neonatal medicine department of the hospital, said. ${ }^{9}$

"This reminds us to pay attention to mother-to-child being a possible route of coronavirus transmission."

It is important to remember that new-born infants can acquire an infection in other ways beyond intrauterine maternal-fetal transmission. In some cases, viral infection can be acquired when the infant passes through the birth canal during a vaginal delivery or breast feeding, although these mechanisms would be highly unusual for a respiratory virus. Neonatal infection from respiratory viruses can occur after delivery through such mechanisms as inhalation of the agent through aerosols produced by coughing from the mother, relatives or healthcare workers or other sources in the hospital environment. Based upon past experience with pregnant women who developed MERS and SARS, and realizing that the numbers are limited, there has never been confirmed intrauterine Coronavirus transmission from mother to fetus. Discussing the most recent baby to be diagnosed with the 2019-nCoV infection, Dr. Stephen Morse, an epidemiologist at the Mailman School of Public Health at Columbia University stated. ${ }^{8}$
"It's more likely that the baby contracted the virus from the hospital environment, the same way healthcare workers get infected by the patients they treat,"

"It's quite possible that the baby picked it up very conventionally-by inhaling virus droplets that came from the mother coughing."

And according to Dr. Paul Hunter, Professor of Medicine at the University of East Anglia. ${ }^{10}$

"As far as I am aware there is currently no evidence that the novel coronavirus can be transmitted in the womb. When a baby is born vaginally it is exposed to the mother's gut microbiome, therefore if a baby does get infected with coronavirus a few days after birth we currently cannot tell if the baby was infected in the womb or during birth."

Given the rapidly progressive spread of this current 2019 novel coronavirus it is reasonable to expect that a significant proportion of pregnant women have already become infected. The effects of 2019-nCoV during pregnancy are, at the present, unknown.

Women during pregnancy often face several pregnancyrelated complications and more susceptible to respiratory pathogens that may put them at a higher risk of adverse consequences. Getting COVID-19 infected at this stage, therefore, may put them at further increased risk of developing adverse pregnancy related and newborn outcomes, including deaths; however, the estimate is lacking. ${ }^{12}$ Similar to the current COVID-19, severe acute respiratory syndrome (SARS) was reported in 2007 and showed devastating consequences if it occurred during pregnancy. ${ }^{11,13}$ Earlier diagnosis of COVID-19 infection status, a clear understanding of all possible symptoms of infection during pregnancy, and proper treatment are important to combat the adverse consequences.

The available studies on the symptoms of COVID-19 were mainly conducted in the general infected people, and fever, cough, fatigue, and shortness of breath were the common symptoms among them. ${ }^{14-19}$ These symptoms reported in a few relevant studies vary for pregnant women, which are inconsistent across studies. For instance, Liu et al identified cough, shortening of breath, and fatigue alongside fever as the most important symptoms of COVID-19 among pregnant women, whereas cough and fever were reported as vital symptoms of COVID-19 in the Zhu et al study. ${ }^{20,21}$ The treatments provided to the COVID-19 infected pregnant women were also varied. Oxygen support, antiviral therapy, and antibiotic therapy were the treatments provided to the infected pregnant women mentioned in Liu et al study. ${ }^{22}$ Alternatively, Zhu et al study reported that antibiotic therapy was avoided for all infected pregnant women, but oxygen support and antiviral therapy were provided. ${ }^{21}$ Under these circumstances, summarizing all possible symptoms and moreover, knowledge of the possible 
adverse outcomes would help healthcare personnel to take precautionary measures earlier.

\section{METHODS}

This study which was carried in SKIMS medical college and hospital, Srinagar, Jammu and Kashmir, India summarizes the pregnancy and perinatal outcomes of women who were infected with COVID- 19 during their pregnancy. It is an observational study. The cases include the pregnant women in their last trimester i.e., third trimester, who were SARS Covid-19 positive, detected by positive RT PCR admitted in the institute from March to June 2020.

\section{Inclusion criteria}

Eligibility criteria included pregnant women in their third trimester positive for covid-19 as detected by real-time PCR or dual fluorescence PCR-confirmed SARS-CoV-2 infection.

\section{Exclusion criteria}

Nonpregnant women positive for COVID-19. Maternal systemic ailments resulting in poor maternal and fetal outcome viz, maternal cardiac ailments included in WHO class III and IV. Poorly controlled diabetes and hypertension. TORCH positive cases. APLA positive cases. Cholestasis of pregnancy were excluded from the study. Outcome of the pregnancies was noted in terms of LSCS rates, low birth weight, preterm delivery, meconium and fetal distress, NICU admissions, maternal and fetal demise and vertical transmission. The cases were followed up to two weeks after termination of pregnancy or discharge from hospital which ever was earlier.

\section{Statistical analysis}

Statistical analysis was done with SPSS, version 25.0 (IBM Corp.). Continuous variables were expressed as mean with standard deviation. Categorical variables were expressed as number of cases and percentages (\%). A pvalue of $\leq 0.05$ was taken as statistically significant.

\section{RESULTS}

Table 1 provides the summary of baseline characteristics and outcome. A total of 38 pregnant women positive for COVID-19 as confirmed by RT-PCR, were included in the study. Mean age of the study group was $27.3 \pm 4$ years. Two of the women had risk factors in the form of diabetes and PIH.

Table 1: Summary of maternal and perinatal characteristics and outcomes.

\begin{tabular}{|c|c|c|c|c|}
\hline \multicolumn{4}{|c|}{ Maternal and perinatal characteristics } & Total \\
\hline \multirow{8}{*}{ Maternal characteristics } & \multirow{2}{*}{ Pregnancy outcome } & Term & Pre term & \\
\hline & & $30(79 \%)$ & $8(21 \%)$ & 38 \\
\hline & \multirow{2}{*}{ Mode of delivery } & LSCS & Vaginal delivery & \\
\hline & & $23(60.5 \%)$ & $15(39.5 \%)$ & 38 \\
\hline & \multirow[b]{2}{*}{ Risk factors } & Diabetes & $\mathrm{PIH}$ & \\
\hline & & 2 & 1 & \\
\hline & Maternal ICU admissions & $1^{* *}$ & & \\
\hline & Maternal deaths & 0 & & \\
\hline \multirow{8}{*}{ Neonatal characteristics } & \multirow{2}{*}{ Apgar score } & Normal & Low & \multirow{2}{*}{38} \\
\hline & & 28 & $10(26.3 \%)$ & \\
\hline & Meconium & $11(29 \%)$ & & \\
\hline & Fetal distress & $11(29 \%)$ & & \\
\hline & Neonatal ICU admissions & $5(13.5 \%)$ & & \\
\hline & Neonatal deaths & $1^{*}(2.7 \%)$ & & \\
\hline & IUD & $1(2.63 \%)$ & & \\
\hline & Vertical transmission & $1(2.6 \%)$ & & \\
\hline \multirow{3}{*}{ Lab characteristics } & Anemia & $8(21 \%)$ & & \\
\hline & Thrombocytopenia & $8(21 \%)$ & & \\
\hline & SARS CoV-2 positive (by RT-PCR) & $38(100 \%)$ & & \\
\hline
\end{tabular}

*Presented with heart failure and shock at $17^{\text {th }}$ day of life. Echo revealed moderate biventricular dysfunction and COVID-19 positive by RT-PCR. Baby also had $6.0 \mathrm{~mm}$ apical muscular VSD, shunting left to right (which could not explain heart failure and biventricular dysfunction). **She had placenta accrete and APH.

Both the diabetic mothers had preterm delivery; however, both the mothers and neonates were discharged in a stable condition. $8(21 \%)$ women had preterm delivery and LSCS (lower segment caesarean section/ C-section) 
was the preferred mode of delivery in 23 of 38 i.e., $60.5 \%$ cases. There was a single maternal ICU admission which was more explained by severe APH owing to her placenta accreta. The mother had IUD (the only IUD in this study).

There was a tendency for low Apgar score at birth with $10(26.3 \%)$ neonates having an Apgar score of less than 7. Fetal distress and meconium was seen in $11(29 \%)$ of the cases. NICU admissions were seen in 5 (13.5\%) of cases and there was one IUD and one neonatal death. The neonate was the only confirmed case of COVID -19 in this study. However, the neonate had tested positive at $17^{\text {th }}$ day of life when he presented with heart failure and ECHO documented biventricular dysfunction and a muscular VSD. Thrombocytopenia - defined as platelet count less than $1.5 \mathrm{lac} / \mathrm{micro}$ litre, was seen in $21 \%$ of cases, however, there was no increased risk of preterm delivery or fetal distress among those with low platelet count.( two preterm births and two meconium positive deliveries i.e., $5.2 \%$ each of preterm births and meconium (incidence of preterm births varies from $5 \%$ to $18 \%$ worldwide as per WHO. In India, the rate of preterm births is estimated at $13 \%$ - UNICEF).

The treatment patterns of COVID-19 infection among pregnant women during their pregnancy or following delivery was mostly supportive in the form of oxygen and antibiotic therapy. No Antiviral therapy was used in these cases. Cephalosporins and macrolides were the commonly used antibiotics.

\section{DISCUSSION}

The world is now experiencing an exponential increase in the COVID-19 infected people, and a significant proportion of them are pregnant women. Though this significant portion of population is especially at high risk of adverse outcome, data is limited in the form of case reports and review articles, on the impact of COVID-19 on this especially vulnerable population. This study is the first of its kind to attempt to address the issue of preterm births, fetal distress and vertical transmission in pregnant women infected by COVID-19 in Indian subcontinent. A total 38 infected pregnant women data were analyzed, and all of them were in the third trimester of pregnancy. There was a tendency for higher preterm births among COVID-19 positive cases. This could be related to stress both psychological and physiological as a result of different interleukins released in response to viral infection. It is pertinent to mention that 5 of 8 preterm births were born by normal vaginal delivery after spontaneous onset of labour, supporting the fact that COVID-19 infection in pregnancy increases the chances of preterm labour.

COVID-19 infection also increases the chances of fetal distress and meconium in liquor, seen in $29 \%$ of cases, which together with increased risk of preterm births increased the incidence of NICU admissions (13.5\%).
One of the important results drawn from the study was absence of evidence of vertical transmission at 48 hours of birth as none of the neonates were detected positive for COVID-19. However, one neonate admitted as heart failure with shock and had a VSD and biventricular dysfunction was detected positive on $17^{\text {th }}$ day of birth and expired after 3 days. The death was attributed to cardiac pump failure in view of moderate biventricular dysfunction which otherwise could not be explained simply by a VSD. This supports the assumption that may be the infection was acquired more conventionally via inhalation or contact with a case or a carrier, however vertical transmission cannot be ruled out and may require follow up for symptoms and may be repeat testing after a couple of weeks.

Oxygen and antibiotic therapy were given as treatments for most infected pregnant women and there was no maternal mortality.

These summary findings will assist healthcare personnel for the better management of pregnant women who have been infected with COVID-19, which has a potential to reduces adverse consequences for women as well as their babies. Several questions have been provoked regarding maternal and neonatal safety if women were infected with COVID-19 during pregnancy. The causes are complications following getting infected and evidence of higher adverse consequences if it has happened with existing morbidity, and these could be added to the usual pregnancy and delivery-related complications. These factors may increase the occurrence of adverse maternal health and birth outcomes, although estimates are lacking. Notably, earlier detection of the infection through tests, symptoms, and effective treatments could reduce these risks.

Given that no specific treatments or vaccines have been discovered yet, the COVID-19 infection during pregnancy would put healthcare providers in additional challenges. $^{22}$ This is because of the possible adverse effects of the medicines used on the fetus as well as pregnancy. For instance, there is evidence that medicines such as chloroquine and hydroxychloroquine are currently recommended for patients with COVID-19, which may cause fetal harm and adversely affect newborns through transferring from mothers' body by breastmilk. $^{23-26}$ Therefore, cautions are needed in the treatment of infected pregnant women.

This study reported, around $60.5 \%$ of the infected mothers had birth reported via use of the $\mathrm{C}$-section; a rate that is significantly higher than the usual WHO's recommendation of $1-5 \%$ use of $\mathrm{C}$-section to avoid death and severe morbidity in mothers and newborns. ${ }^{27,28}$ Getting COVID-19 infection increases complex viral infection among women in pregnancy; therefore, Csection is recommended to reduce perinatal and neonatal adverse outcomes. ${ }^{29}$ Earlier preparedness of the healthcare sectors to handle these adverse consequences 
would be helpful to reduce further adverse outcomes, including maternal and perinatal mortality.

This study has several strengths and limitations, this was the first study of its kind that highlight the pregnancy and perinatal outcomes among women who have been infected with COVID-19. The major limitations being an observational study and small sample size.

Despite these limitations, this study has enough merits, which will make healthcare providers aware of the possible outcomes in pregnant women detected with COVID-19.

\section{CONCLUSION}

This study confirms that COVID-19 infection during pregnancy increases the risk of several adverse outcomes, including higher rates of caesarean delivery, low birth weight, and preterm birth, fetal distress, NICU admission rate. While the vertical transmission could not be confirmed, it cannot be ruled out as well. Healthcare providers may consider these for effective management of COVID-19 infected pregnant women, which would reduce pregnancy-related adverse consequences, including maternal and new-born morbidity and mortality.

\section{Funding: No funding sources} Conflict of interest: None declared

Ethical approval: The study was approved by the Institutional Ethics Committee

\section{REFERENCES}

1. Li Q, Guan X, Wu, P, Wang X, Zhou L, Tong Y, et al. Early transmission dynamics in Wuhan, China, of novel coronavirus-infected pneumonia. N Engl J Med. 2020;382:1199-207.

2. Zhu N, Zhang D, Wang W, Li X, Yang B, Song J, et al. A novel coronavirus from patients with pneumonia in China, 2019. N Engl J Med. 2020;382:727-33.

3. Wong SF, Chow KM, Leung TN, Ng WF, Ng TK, Shek CC, et al. Pregnancy and perinatal outcomes of women with severe acute respiratory syndrome. Am J Obstet Gynecol. 2004;191:292-7.

4. Alfaraj SH, Al-Tawfiq JA, Memish ZA. Middle East respiratory syndrome coronavirus (MERS-CoV) infection during pregnancy: report of two cases and review of the literature. J Microbiol Immunol Infect. 2019;52:501-3.

5. O'Day MP. Cardio-respiratory physiological adaptation of pregnancy. Semin Perinatol. 1997;21:268-75.

6. Tsafaras GP, Ntontsi P, Xanthou G. Advantages and limitations of the neonatal immune system. Front Pediatr. 2020;8:5.

7. Steinbuch, Y. Chinese baby tests positive for coronavirus 30 hours after birth. Available at: https://nypost.com/2020/02/05/chinese-baby-testspositive-for-coronavirus-30-hours-after-birth/. Accessed on $9^{\text {th }}$ February 2020.

8. Woodward, A. A pregnant mother infected with the coronavirus gave birth, and her baby tested positive 30 hours later. Available at: https://www.businessinsider.com/wuhan-coronavirus -in-infant-bornfrom-infected-mother-2020-2. Accessed on $8^{\text {th }}$ February 2020.

9. Gillespie, T. Coronavirus: doctors fear pregnant women can pass on illness after newborn baby is diagnosed. Available at: https://news.sky.com/story/coronavirus-doctors-fearpregnant-women-canpass- on-illness-after-newbornbaby-is-diagnosed-11926968. Accessed on $8^{\text {th }}$ February 2020.

10. Science Media Centre. Expert Reaction to Newborn Baby Testing Positive for Coronavirus in Wuhan. Available at: https://www.sciencemediacentre.org/ expert-reaction-to-newborn-baby-testing-positiveforcoronavirus-in-wuhan/. Accessed on $9^{\text {th }}$ February 2020.

11. Zhang S, Diao M, Yu W, Pei L, Lin Z, Chen D. Estimation of the reproductive number of novel coronavirus (COVID-19) and the probable outbreak size on the Diamond Princess cruise ship: A datadriven analysis. Int J Infect Dis. 2020;93:201-4.

12. Liu H, Wang LL, Zhao SJ, Kwak-Kim J, Mor G, Liao AH. Why are pregnant women susceptible to viral infection: an immunological viewpoint?. J Reprod Immunol. 2020:103122.

13. Wong SF, Chow KM, Leung TN, Ng WF, Ng TK, Shek CC, et al. Pregnancy and perinatal outcomes of women with severe acute respiratory syndrome. Am J Obstet Gynecol. 2004;191(1):292-7.

14. Wang D, Hu B, Hu C, Zhu F, Liu X, Zhang J, et al. Clinical characteristics of 138 hospitalized patients with 2019 novel coronavirus-infected pneumonia in Wuhan, China. JAMA. 2020;323(11):1061-9.

15. Bastola A, Sah R, Rodriguez-Morales AJ, Lal BK, Jha R, Ojha HC, et al., The first 2019 novel coronavirus case in Nepal. Lancet Infect Dis. 2020;20(3):279-80.

16. Cheng SC, Chang YC, Chiang YL, Chien YC, Cheng M, Yang CH, et al. First case of Coronavirus disease 2019 (COVID-19) pneumonia in Taiwan. J Formosan Med Assoc. 2020;119(3):747-51.

17. Holshue ML, DeBolt C, Lindquist S, Lofy KH, Wiesman J, Bruce H, et al. First case of 2019 novel coronavirus in the United States. New Engl J Med. 2020;382(10):929-36.

18. Kim JY, Choe PG, Oh Y, Oh KJ, Kim J, Park SJ, et al. The first case of 2019 novel coronavirus pneumonia imported into Korea from Wuhan, China: implication for infection prevention and control measures. J Korean Med Sci. 2020;35(5).

19. Silverstein WK Stroud L, Cleghorn GE, Leis JA. First imported case of 2019 novel coronavirus in Canada, presenting as mild pneumonia. Lancet. 2020;395(10225):734. 
20. Liu H, Liu F, Li J, Zhang T, Wang D, Lan W. Clinical and CT imaging features of the COVID-19 pneumonia: focus on pregnant women and children. $\mathrm{J}$ Infect. 2020;11:11.

21. Zhu H, Wang L, Fang C, Peng S, Zhang L, Chang G, et al. Clinical analysis of 10 neonates born to mothers with 2019-nCoV pneumonia. Translat Pediatr. 2020;9(1):51-60.

22. Liu D, Li L, Wu X, Zheng D, Wang J, Yang L, et al. Pregnancy and perinatal outcomes of women with coronavirus disease (COVID-19) pneumonia: a preliminary analysis. Am J Roentgenol. 2020:1-6.

23. Gautret P, Lagier JC, Parola P, Meddeb L, Mailhe M, Doudier B, et al. Hydroxychloroquine and azithromycin as a treatment of COVID-19: results of an open-label non-randomized clinical trial. Int J Antimicrobial Agents. 2020:105949.

24. Levy M, Buskila D, Gladman DD, Urowitz MB, Koren G. Pregnancy outcome following first trimester exposure to chloroquine. Am J Perinatol. 1991;8(3):174-8.

25. American academy of pediatrics committee on drugs. Transfer of drugs and other chemicals into human milk. Pediatr. 2001;108(3):776.
26. Boelaert JR, Yaro S, Augustijns P, Meda N, Schneider YJ, Schols D, et al. Chloroquine accumulates in breast-milk cells: potential impact in the prophylaxis of postnatal mother-to-child transmission of HIV-1. Aids. 2001;15(16):2205-7.

27. De Brouwere V, Dubourg D, Richard F, Van Lerberghe W. Need for caesarean sections in west Africa. Lancet. 2002;359(9310):974-5.

28. Khan MN, Islam MM, Shariff AA, Alam MM, Rahman MM. Socio-demographic predictors and average annual rates of caesarean section in Bangladesh between 2004 and 2014. PloS One. 2017;12(5):e0177579.

29. Sharma D, Spearman P, The impact of cesarean delivery on transmission of infectious agents to the neonate. Clin Perinatol. 2008;35(2):407-20.

Cite this article as: Hassan N, Muzamil M, Banday D. COVID-19 infection during pregnancy - maternal and perinatal outcomes: a tertiary care centre study. Int J Reprod Contracept Obstet Gynecol 2020;9:3764-9. 